\title{
Terrorismus
}

\section{Die Persönlichkeit von Attentätern}

In Zeiten des Terrors stellen sich viele die Frage: Was macht Menschen zu Attentätern? Psychisch krank sind die wenigsten, meinen einige Psychiater. Sie haben die Persönlichkeiten von extremistischen Gewalttätern analysiert - und mehrere klassische Täterprofile ausfindig gemacht.

Lassen sich gewaltbereite Extremisten schon vor der Tat identifizieren? Auf dem vergangenen DGPPN-Kongress in Berlin wurden hier unterschiedliche Betrachtungsweisen deutlich. Auf der einen Seite betonen Psychiater immer wieder, dass extremistische Gewalttaten zum großen Teil von psychisch Gesunden begangen werden, die ein abweichendes Wertegefühl entwickelt haben. Die Radikalisierung sehen sie vor allem als sozialen Prozess und weniger als Ergebnis einer gestörten Psyche oder Persönlichkeit. "Es gibt kein typisches Persönlichkeitsmuster, das es erlauben würde, die Täter frühzeitig zu identifizieren", sagte Kongresspräsidentin Prof. Dr. Iris Hauth.

Andere hingegen betonten durchaus die Bedeutung der Attentäterpersönlichkeit. So sieht Prof. Dr. Jérôme Endrass, Konstanz, kaum Unterschiede zwischen extremistischen Attentätern und anderen Gewaltverbrechern. Die Ideologie sei oft austauschbar und nachrangig, im Vordergrund stehe bei einem Teil der Täter die Gewaltbereitschaft.

\section{Radikale müssen nicht}

\section{gewalttätig sein}

Die Radikalisierung hält Endrass nur für eine von mehreren Dimensionen extremistischer Gewalttäter. Man sollte sie bei der Risikobewertung nicht zu stark gewichten: "Es gibt viele Menschen mit radikalen Vorstellungen, aber längst nicht jeder von ihnen wird gewalttätig."

A Nach Auffassung von Endrass lässt sich der Kreis der gewaltbereiten Extremisten aber deutlich einschränken, wenn neben der Radikalität bestimmte Persönlichkeitsmerkmale berücksichtigt werden. Dazu zählt er Dissozialität, martialisches Auftreten, Waffenaffinität, Wahnvorstellungen, Substanzabusus sowie latente oder akute Suizidalität. Eine weitere Di- mension sieht er im Warnverhalten, etwa wenn Personen im Internet Drohungen verkünden. Schließlich seien Kontext, Lebenssituation und Belastungsfaktoren $\mathrm{zu}$ berücksichtigen. Geraten potenzielle Täter nach entsprechenden Drohungen unter Zugzwang? Befinden sie sich in einer persönlichen Krise? Spitzt sich ihre soziale Situation aufgrund finanzieller oder sozialer Probleme zu?

\section{Drei Prototypen}

Nach Auffassung des Psychologen lassen sich drei Prototypen von Attentätern ableiten:

Beim ersten Typ sind gesellschaftliche Regeln und Normen nicht verankert. Solche Personen halten Gewaltanwendung generell für legitim und benötigen dafür keinen ideologischen Überbau. Sie sind häufig dissozial, impulsiv, aufbrausend und in der Vergangenheit durch Straftaten auffällig geworden. Für sie spielt die Radikalisierung eine eher geringe Rolle, sie dient eher der Legitimierung von Gewalttaten.

Beim zweiten Typ sind gesellschaftliche Regeln und Normen nur kontextspezifisch verankert, sie gelten zum Beispiel nicht für Andersgläubige. Diese darf man daher töten. Um zu dieser Überzeugung zu gelangen, ist eine bedeutsame "Legitimierungsarbeit" nötig. Für diesen Typ ist die Ideologie sehr wichtig. Sie hilft ihm, die verankerten Normen für bestimmte Gruppen außer Kraft zu setzen. Die Radikalisierung ist daher von großer Bedeutung. Solche Personen leben wie Syed Farook häufig in einer stabilen Beziehung und sind bisher nicht strafrechtlich aufgefallen.

Beim dritten Typ sind gesellschaftliche Regeln und Normen gut verankert, allerdings ist die Realitätswahrnehmung durch eine psychische Störung getrübt. Solche
Personen waren in der Vergangenheit häufig psychiatrisch auffällig oder hatten Drogenprobleme. Sie werden in der Regel nur dann gewalttätig, wenn sie ausgeprägte Krankheitssymptome zeigen. Dies war etwa der Fall beim Attentäter, der 1990 auf Wolfgang Schäuble schoss.

\section{Wege in die Radikalisierung}

Radikalisierung ist eine Voraussetzung für extremistische Gewalt. Vor allem bei Personen, die nicht zum Kreis notorischer Gewalttäter gehören, ist im Laufe des Radikalisierungsprozesses eine intensive kognitive „Legitimierungsarbeit“ nötig. Um diese zu verstehen, sind für Prof. Dr. Jérôme Endrass auch Erkenntnisse aus der forensischen Psychologie hilfreich, etwa zu Sexualstraftätern. Diese würden mit der Zeit „implizite Theorien“ entwickeln, um ihr Handeln auch vor sich selbst zu rechtfertigen, etwa: „Kinder provozieren Sex mit Erwachsenen“ oder "Sexualität ist nicht kontrollierbar".

In gewisser Weise sei dies auch eine Form der Radikalisierung. Solche Denkweisen werden vor allem dann relevant, wenn sie sich langsam entwickeln, wenn Erfahrungen mit der Gesellschaft mit einfließen und sich dabei die eigenen Wert- und Normvorstellungen verändern. Dann entstehen daraus „kognitive Gepflogenheiten“.

Übertragen auf den radikalen Islamismus wäre eine solche Gepflogenheit etwa die Überzeugung, Andersgläubige töten zu müssen. Anfangs könnte zunächst die implizite Theorie stehen, „Muslime werden unterdrückt“, im weiteren Verlauf folgen Überzeugungen wie „Muslime müssen sich in einer feindlich gesinnten Welt behaupten“. Parallel dazu verändern sich die Normen: Anfangs steht noch ein Bekenntnis $\mathrm{zu}$ den rechtsstaatlichen Prinzipien, später wird die Scharia als gleichwertig betrachtet, schließlich wird die Scharia herangezogen, um die Vernichtung der Feinde zu begründen. 